\title{
Analysis of cerebrovascular aneurysm treatment cost: retrospective cohort comparison of clipping, coiling, and flow diversion
}

\author{
*Spencer Twitchell, BS, Hussam Abou-Al-Shaar, MD, Jared Reese, BS, \\ Michael Karsy, MD, PhD, MSc, llyas M. Eli, MD, Jian Guan, MD, Philipp Taussky, MD, and \\ William T. Couldwell, MD, PhD
}

Department of Neurosurgery, Clinical Neurosciences Center, University of Utah, Salt Lake City, Utah

\begin{abstract}
OBJECTIVE With the continuous rise of health care costs, hospitals and health care providers must find ways to reduce costs while maintaining high-quality care. Comparing surgical and endovascular treatment of intracranial aneurysms may offer direction in reducing health care costs. The Value-Driven Outcomes (VDO) database at the University of Utah identifies cost drivers and tracks changes over time. In this study, the authors evaluate specific cost drivers for surgical clipping and endovascular management (i.e., coil embolization and flow diversion) of both ruptured and unruptured intracranial aneurysms using the VDO system.
\end{abstract}

METHODS The authors retrospectively reviewed surgical and endovascular treatment of ruptured and unruptured intracranial aneurysms from July 2011 to January 2017. Total cost (as a percentage of each patient's cost to the system), subcategory costs, and potential cost drivers were evaluated and analyzed.

RESULTS A total of 514 aneurysms in 469 patients were treated; 273 aneurysms were surgically clipped, 102 were repaired with coiling, and 139 were addressed with flow diverter placements. Middle cerebral artery aneurysms accounted for the largest portion of cases in the clipping group (29.7\%), whereas anterior communicating artery aneurysms were most frequently involved in the coiling group (30.4\%) and internal carotid artery aneurysms were the majority in the flow diverter group (63.3\%). Coiling (mean total cost $0.25 \% \pm 0.20 \%$ ) had a higher cost than flow diversion (mean $0.20 \% \pm$ $0.16 \%$ ) and clipping (mean $0.17 \pm 0.14 \% ; p=0.0001$, 1-way ANOVA). Coiling cases cost 1.5 times as much as clipping and flow diversion costs 1.2 times as much as clipping. Facility costs were the most significant contributor to intracranial clipping costs $(60.2 \%)$, followed by supplies (18.3\%). Supplies were the greatest cost contributor to coiling costs $(43.2 \%)$, followed by facility (40.0\%); similarly, supplies were the greatest portion of costs in flow diversion $(57.5 \%)$, followed by facility (28.5\%). Cost differences for aneurysm location, rupture status, American Society of Anesthesiologists (ASA) grade, and discharge disposition could be identified, with variability depending on surgical procedure. A multivariate analysis showed that rupture status, surgical procedure type, ASA status, discharge disposition, and year of surgery all significantly affected cost $(p<0.0001)$.

CONCLUSIONS Facility utilization and supplies constitute the majority of total costs in aneurysm treatment strategies, but significant variation exists depending on surgical approach, rupture status, and patient discharge disposition. Developing and implementing approaches and protocols to improve resource utilization are important in reducing costs while maintaining high-quality patient care.

https://thejns.org/doi/abs/10.3171/2018.1.FOCUS17775

KEYWORDS cost-effectiveness; aneurysm; clipping; coiling; flow diversion; value-driven outcome

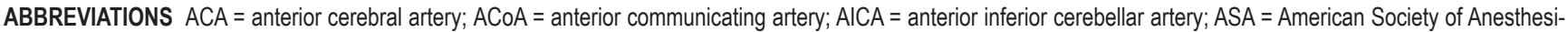
ologists; $\mathrm{BA}=$ basilar artery; ICA = internal carotid artery; $\mathrm{LOS}=$ length of stay; $\mathrm{MCA}=$ middle cerebral artery; PCA = posterior cerebral artery; PCoA = posterior communicating artery; PICA = posterior inferior cerebellar artery; SCA = superior cerebellar artery; STROBE = Strengthening the Reporting of Observational Studies in Epidemiology; $\mathrm{VA}=$ vertebral artery; $\mathrm{VDO}=$ Value-Driven Outcomes.
}

SUBMITTED December 19, 2017. ACCEPTED January 15, 2018.

INCLUDE WHEN CITING DOI: 10.3171/2018.1.FOCUS17775.

* S.T. and H.A.A.S. contributed equally to this work. 
$\mathrm{T}$ HE health care system costs and quality of care in the US currently represent a vital issue to the American people. The importance of delivering better care at a lower cost has been the objective for Americans for the past two decades. It is estimated that Americans spend \$2.7 trillion a year on health care alone, constituting $18 \%$ of the gross domestic product of the country. ${ }^{19}$ Interestingly, it is estimated that $30 \%$ of that cost is wasted on unnecessary and costly services. The importance of this issue increases the demand on providers to limit expenditures and avoid cost-ineffective treatment modalities.

The University of Utah has implemented the ValueDriven Outcomes (VDO) tool to analyze patient costs, evaluate cost drivers, and find ways to reduce cost expenditures while maintaining a high quality of care. ${ }^{9,11}$ The features of this system have aided us in evaluating obscured care costs, patients' quality of life, and hidden cost drivers of various neurosurgical procedures. ${ }^{2,7,14}$ We previously reported the efficacy of the VDO system in evaluating the impact of various factors on the cost of treatment for patients with pituitary tumors. ${ }^{7}$

The advancement of interventional techniques in the management of intracranial aneurysms and the results of the International Subarachnoid Hemorrhage Trial have convinced many neurosurgeons to switch their management strategy from the surgical to the endovascular route. ${ }^{15}$ However, the costs of surgical versus endovascular intervention as well as the cost-effectiveness of these two approaches have been an area of debate since the late 1990s, with various studies showing contradictory results. In addition, the increased use of flow diverter devices for complex aneurysm treatment has affected the cost of care for patients treated for aneurysms. Therefore, the aim of this study was to evaluate specific cost drivers for surgical clipping and endovascular management (i.e., coil embolization and flow diversion) of both ruptured and unruptured intracranial aneurysms using the VDO system.

\section{Methods}

The study was approved by the IRB at the University of Utah. The data were collected using the VDO database and the electronic medical record database to isolate elective and emergent patient cases in which aneurysms were treated via intracranial clipping, coiling, or flow diverter placement.

\section{The VDO Database}

The VDO database is a novel resource that generates direct costs in lieu of patient/insurer charges, and its methodology has been previously reported. ${ }^{7}$ To identify patients for this study, the common procedural terminology codes 61624 (endovascular therapy procedures on skull, meninges, and brain), 61697 (complex intracranial aneurysm carotid circulation), 61698 (complex intracranial aneurysm vertebrobasilar circulation), 61700 (simple intracranial aneurysm carotid circulation), and 61702 (simple intracranial aneurysm vertebrobasilar circulation) were searched for the period from July 2011 to January
2017, during which cost data were available. All available cost data at the time of the index surgical treatment, from admission to discharge, were evaluated. After review of the individual patient charts, only patients with confirmed intracranial aneurysm repairs using clipping, coiling, or flow diversion (i.e., flow diverter placement) were included. All aneurysm locations were collected, along with the demographic and clinical data for the patient. Various skull base approaches were utilized in the clipping group depending on aneurysm location and the preference of the senior authors (P.T. and W.T.C.). At our institution, a multidisciplinary neurovascular conference is held weekly where complex cases are discussed by the providers prior to selection of a treatment modality.

\section{Cost Variables}

The total cost and subcategory costs were analyzed, including pharmacy, operating room/surgical services, intensive care unit, acute care, imaging, supplies and implants, laboratory, food services, therapies, emergency department, and facility costs. Subcategory cost is reported as a percentage of total cost, and actual dollar amounts are not reported as per agreement with the university. Costs for aneurysm repair equipment and supplies were regularly discussed by faculty to improve uniformity of practice management.

Mean percentage of total cost was generated to overcome the inability to present actual cost data out of contractual obligation with our hospital. For the entire cohort of patients, costs were summed and the fraction of total cost contribution for each patient was calculated. Thus, means, standard deviations, and comparison of patient total and subcategory costs could be assessed. Mean percentage of total costs may not necessarily total 100\%. For subcategory cost contribution, each patient's subcategory cost was divided by the total. For these calculations, percentages will equal $100 \%$.

\section{Statistical Analysis}

Variable means and standard deviations were calculated where appropriate. Continuous variables were analyzed via t-test. Discrete variables were analyzed using the chi-square test. A multivariable regression analysis was used to evaluate factors that affected cost. All statistical analyses were performed using IBM SPSS Statistics for Windows (version 20.0, IBM Corp.) with $\mathrm{p}<0.05$ considered statistically significant. The STROBE (Strengthening the Reporting of Observational Studies in Epidemiology) checklist was utilized in the preparation of this paper (https://www.strobe-statement.org/).

\section{Results}

\section{Patient Demographics and Procedures}

A total of 514 aneurysms in 469 patients were treated from July 2011 to January 2017. Among those, 273 were surgically clipped, 102 were treated with coiling, and 139 were addressed with flow diverter placement (Table 1). There was a significant difference in mean age among the groups, with the lowest age found in the clipping group $(52.3 \pm 13.3$ years $)$ followed by flow diversion $(56.0 \pm 14.7$ 
TABLE 1. Patient demographics

\begin{tabular}{|c|c|c|c|c|}
\hline Variable & Clipping $(n=273)$ & Coil Embolization $(n=102)$ & Flow Diversion $(n=139)$ & $\mathrm{p}$ Value \\
\hline Mean age $\pm S D$, yrs & $52.3 \pm 13.3$ & $57.6 \pm 13.3$ & $56.0 \pm 14.7$ & 0.006 \\
\hline Aneurysm location, no. (\%) & & & & 0.0001 \\
\hline ICA & $55(20.1)$ & $12(11.8)$ & $88(63.3)$ & \\
\hline ACA & $17(6.2)$ & $17(16.7)$ & $5(3.6)$ & \\
\hline $\mathrm{ACoA}$ & $65(23.8)$ & $31(30.4)$ & $2(1.4)$ & \\
\hline PCoA & $20(7.3)$ & $8(7.8)$ & $5(3.6)$ & \\
\hline MCA & $81(29.7)$ & $1(1.0)$ & $5(3.6)$ & \\
\hline $\mathrm{BA}$ & $6(2.2)$ & $20(19.6)$ & $8(5.8)$ & \\
\hline VA/SCA/PCA/PICA/AICA & $13(4.8)$ & $8(7.8)$ & $24(17.3)$ & \\
\hline Multiple & $16(5.9)$ & $5(4.9)$ & $2(1.4)$ & \\
\hline Aneurysm status, no. (\%) & & & & 0.0001 \\
\hline Unruptured & $129(47.3)$ & $41(40.2)$ & $134(96.4)$ & \\
\hline Ruptured & $144(52.7)$ & $61(59.8)$ & $5(3.6)$ & \\
\hline Male sex, no. (\%) & $104(38.1)$ & $38(37.3)$ & $32(23.0)$ & 0.007 \\
\hline ASA grade, no. (\%) & & & & 0.0001 \\
\hline Unknown & $17(6.2)$ & $55(53.9)$ & $59(42.5)$ & \\
\hline 1 & $4(1.5)$ & $2(2.0)$ & $1(0.7)$ & \\
\hline 2 & $57(20.9)$ & $6(5.9)$ & $31(22.3)$ & \\
\hline 3 & $117(42.9)$ & $17(16.7)$ & $32(23.0)$ & \\
\hline 4 & $65(23.8)$ & $19(18.6)$ & $13(9.4)$ & \\
\hline 5 & $8(2.9)$ & $3(2.9)$ & $3(2.2)$ & \\
\hline 6 & $5(1.8)$ & $0(0.0)$ & $0(0.0)$ & \\
\hline Mean LOS \pm SD, days & $11.7 \pm 9.7$ & $10.5 \pm 10.2$ & $4.4 \pm 7.1$ & 0.0001 \\
\hline Discharge disposition, no. (\%) & & & & 0.0001 \\
\hline Home/self-care & $147(53.8)$ & $62(60.8)$ & $117(84.2)$ & \\
\hline Home health services & $14(5.1)$ & $2(2.0)$ & $6(4.3)$ & \\
\hline Acute rehabilitation & 74 (27.1) & $16(15.7)$ & $7(5.0)$ & \\
\hline Long-term acute care & $7(2.6)$ & $4(3.9)$ & $2(1.4)$ & \\
\hline Skilled nursing facility & $13(4.8)$ & $7(6.9)$ & $3(2.2)$ & \\
\hline Deceased & $18(6.6)$ & $11(10.8)$ & $4(2.9)$ & \\
\hline Mean total cost \pm SD, $\%$ & $0.17 \pm 0.14$ & $0.25 \pm 0.20$ & $0.20 \pm 0.16$ & 0.0001 \\
\hline
\end{tabular}

years) and coiling $(57.6 \pm 13.3 ; \mathrm{p}=0.006)$. A significant difference in sex was also noted, among the clipping (38.1\% male), coiling (37.3\% male), and flow diversion (23.0\% male) groups $(\mathrm{p}=0.007)$.

Middle cerebral artery (MCA) aneurysms accounted for the largest number of cases $(29.7 \%)$ in the clipping group, followed by anterior communicating artery (ACoA) aneurysms (23.8\%) and internal carotid artery (ICA) aneurysms (20.1\%). In the coiling group, the ACoA was the most common location for the aneurysm (30.4\%), followed by the basilar artery (BA; 19.6\%), whereas the ICA contained the majority (63.3\%) of aneurysms in the flow diverter group, followed by vertebral artery (VA)/superior cerebellar artery (SCA)/posterior cerebral artery (PCA)/posterior inferior cerebellar artery (PICA)/anterior inferior cerebellar artery (AICA) locations (17.3\%).

The surgical clipping group had a mean hospital length of stay (LOS) of $11.7 \pm 9.7$ days, whereas the coiling group had a mean LOS of $10.5 \pm 10.2$ days and the flow diverter placement group had a mean LOS of $4.4 \pm 7.1$ days. Of the clipped, coiled, and flow diverter-treated aneurysms, $52.7 \%, 59.8 \%$, and $3.6 \%$ presented after rupture, respectively.

\section{Total Costs}

Average costs between clipping, coiling, and flow diversion were significantly different $(p=0.0001$, one-way ANOVA). Intracranial aneurysm surgical clipping had a mean percentage of total cost of $0.17 \% \pm 0.14 \%$, coiling had a mean percentage of total cost of $0.25 \% \pm 0.20 \%$, and flow diversion had a mean percentage of total cost of $0.20 \% \pm 0.16 \%$. Thus, on average, coiling cost 1.5 times as much as clipping and flow diverters cost 1.2 times as much as clipping.

\section{Cost Subcategory Analysis}

Subcategory costs were broken down by aneurysm treatment modality (Fig. 1). For intracranial clipping, facil- 

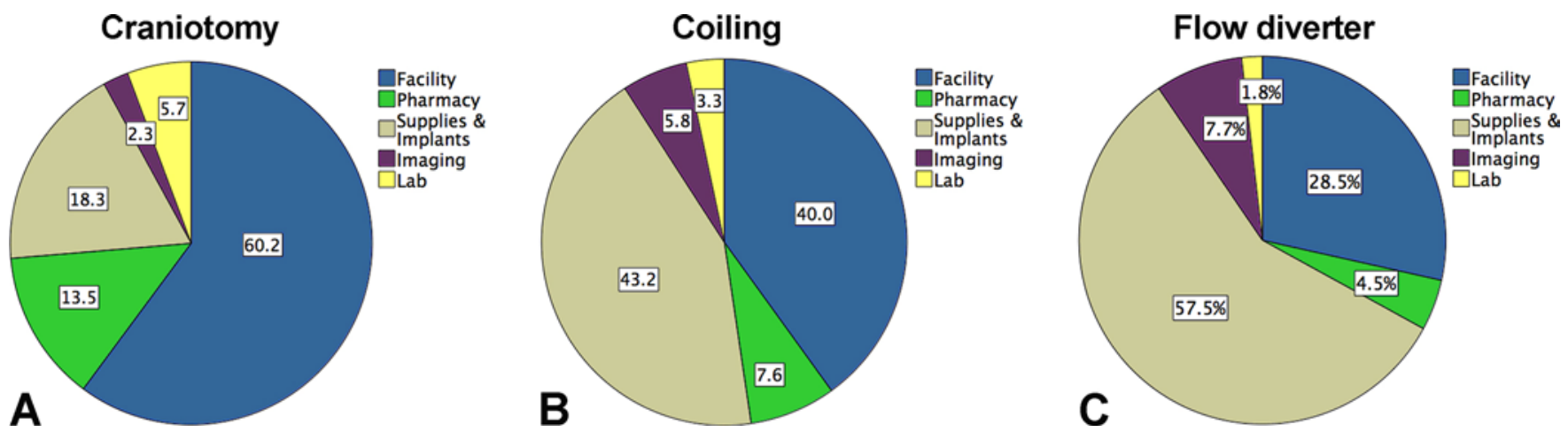

FIG. 1. Cost breakdown for open surgical clipping (A), endovascular coiling (B), and endovascular flow diversion (C) is shown for facility, supplies and implants, pharmacy, imaging, and laboratory costs. Facility charges were the largest contributor $(60.2 \%)$ to surgical clipping costs, whereas supplies were the largest contributor to coiling (43.2\%) and flow diversion $(57.5 \%)$ costs. There were significant differences in facility, pharmacy, supply, imaging, and laboratory cost distributions among the three procedure types $(p<0.0001$, chi-square test).

ity costs were the most significant contributor $(60.2 \%)$, followed by supplies (18.3\%), pharmacy (13.5\%), laboratory $(5.7 \%)$, and imaging (2.3\%). Supplies were the greatest contributor to costs for coiling procedures $(43.2 \%)$, followed by facility $(40.0 \%)$, pharmacy $(7.6 \%)$, imaging $(5.8 \%)$, and laboratory (3.3\%). Cost contributors for flow diverter placement were similar, as supplies were the greatest influence on total cost (57.5\%), followed by facility (28.5\%), imaging (7.7\%), pharmacy $(4.5 \%)$, and laboratory $(1.8 \%)$. Overall, there was a significant difference in cost subtotals among different treatment types $(\mathrm{p}<0.0001$, chi-square test).

The influence of time on the total cost was analyzed (Fig. 2A). From 2011 to 2017, there was significant variability in cost for all 3 surgical procedures. Coiling and flow diverter treatments were biased toward being more recent procedures because of the more recent adoption of these techniques at our institution. The costs of clipping (standardized $\beta=0.15, \mathrm{p}=0.02)$, coiling $(\beta=0.14, \mathrm{p}=$ $0.16)$, and flow diversion $(\beta=0.26, p=0.002)$ all increased over time, with flow diverter procedure costs increasing at the highest rate. Figure $2 \mathrm{~B}$ compares the relative contribution of each subtype cost for each of the procedure types. Initial analysis of procedural costs based on aneurysm location suggested that vertebrobasilar aneurysms had the highest cost of treatment regardless of treatment approach (Fig. 2C). Ruptured aneurysms cost 2.6, 2.3, and 2.7 times more to treat compared with elective cases for clipping, coiling, and flow diversion, respectively (Fig. 2D). Higher American Society of Anesthesiologists (ASA) grade correlated with higher overall treatment cost for all treatment types (Fig. 2E). In addition, discharge dispositions other than home/self-care or home health resulted in increased treatment costs (Fig. 2F).

The potential cost drivers were evaluated with univariate and multivariable regression analysis (Table 2). Using significant factors from the univariate analysis, multivariable analysis showed that aneurysm rupture status $(\mathrm{p}$ $=0.0001)$, procedure type $(\mathrm{p}=0.0001)$, ASA grade $(\mathrm{p}=$ $0.0001)$, discharge disposition $(\mathrm{p}=0.0001)$, and year of operation $(\mathrm{p}=0.0001)$ all contributed to the total costs while aneurysm location $(\mathrm{p}=0.5)$ and $\operatorname{sex}(\mathrm{p}=0.2)$ did not affect cost.

\section{Discussion}

\section{Study Findings}

We have delineated important factors that contribute to the total costs for patients undergoing aneurysm treatment. A total of 514 aneurysms in 469 patients were treated by clipping ( $\mathrm{n}=273)$, coiling $(\mathrm{n}=102)$, or flow diverter placement $(n=139)$ over a 6-year period. Facility costs were predominant in clipping while supplies and implants were the largest expenses in coiling and flow diversion. Coiling cost an average of 1.5 times more than clipping, and flow diverters cost 1.2 times more than clipping. Ruptured aneurysms, higher ASA grade, and non-home/self-care or home health discharge dispositions resulted in increased treatment costs. A multivariate analysis confirmed that rupture status, procedure type, ASA grade, discharge disposition, and year of operation all affected cost.

\section{Aneurysm Treatment Cost}

Previous studies have sought to examine the cost of aneurysm treatment. Bairstow et al. ${ }^{1}$ compared the costs and outcomes of surgical clipping and endovascular coiling in the treatment of ruptured intracranial aneurysms. They found that endovascular procedures tended to be shorter in duration, with lower staffing costs, higher consumables costs, higher total costs, and shorter LOS. Familiari et al. ${ }^{4}$ reported similar LOS between surgically and endovascularly (coil embolization and flow diversion) treated patients with giant unruptured aneurysms, but there was no significant difference in facility costs (intensive care unit and ward) between the two groups. The study did indicate that there was a higher supply cost in the endovascularly treated arm compared with the surgical clipping arm. In our series, we observed a higher facility cost in the surgical group and higher supply costs for the coiling and flow diverter groups. In the study by Familiari et al., ${ }^{4}$ imaging made up $3.1 \%$ of costs in the endovascular group and $14.0 \%$ of costs in the clipping group, hospital stay was $28.7 \%$ of costs in the endovascular group and $51.2 \%$ of costs in the surgical group, and implants made up 39.9\% of costs in the endovascular group and $0.8 \%$ of costs in the surgical clipping group. ${ }^{4}$ Our findings were similar to 

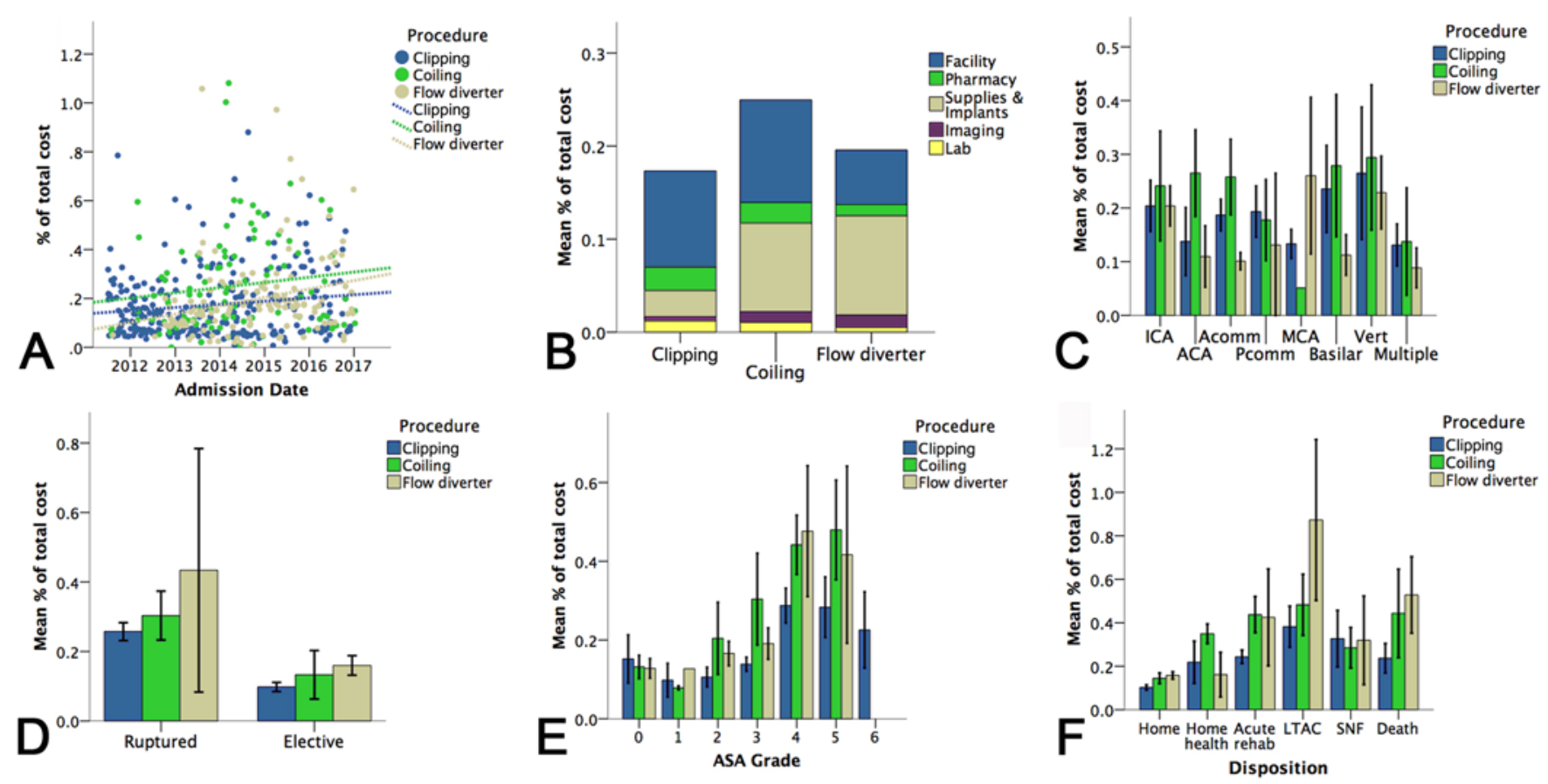

FIG. 2. Evaluation of costs during treatment of aneurysms with clipping, coiling, and flow diverter placement. A: Cost variability over time is shown for clipping, coiling, and flow diversion cases. Although there was significant variability, only a slight increase in cost over time is appreciated. Increased used of coiling and flow diversion procedures is observed in later years compared with clipping procedures. B: The average relative contribution of each subtype cost for each procedure type is illustrated. Coiling procedures were, on average, more expensive than flow diversion and clipping cases. Relative differences in subtotal costs can be appreciated. C: The illustration of cost for different aneurysm locations by procedure type suggests aneurysms in the BA (Basilar) and VA (Vert) are the most expensive to treat. $A C A=$ anterior cerebral artery; $A C o m m=A C O A ; P c o m m=$ posterior communicating artery (PCoA). D: Cost for ruptured aneurysm treatment was significantly higher than for elective treatment by clipping ( $p$ $=0.0001)$, coiling $(p=0.001)$, and flow diversion $(p=0.0001)$. E: Higher costs were associated with higher ASA grade for all procedures. F: Patient discharge disposition to rehabilitation, long-term acute care (LTAC), or skilled nursing facility (SNF) locations involved higher costs, likely as a result of longer hospital stays and increased acuity, compared with home and home health discharge dispositions.

those of Bairstow et al. and Familiari et al., as we also demonstrated that endovascular intervention is more expensive than surgical clipping. Other groups have also observed the more costly treatment of aneurysms using endovascular therapy. ${ }^{6,10,12}$

Colby and colleagues ${ }^{3}$ compared the costs of coiling and flow diverters in the management of anterior circulation aneurysms and found that total costs (equipment plus implants) were significantly lower in the flow diverter arm compared with the stent coiling arm $(\mathrm{p}=0.02)$. Interestingly, it has been reported that as the size of an aneurysm increases, the cost of coils and flow diverters increases dramatically as well. This has been attributed to the increased utilization of more coils/flow diverters to completely pack the aneurysm. For instance, Simon et al.$^{18}$ found the cost of coils for a 3-mm aneurysm ranged from $\$ 599$ to $\$ 3040$ while the cost for a $25-\mathrm{mm}$ aneurysm ranged from $\$ 32,873$ to $\$ 357,095$, representing a 54.9 - to 117.5 -fold increase in cost for an 8.3-fold increase in aneurysm diameter. We did not specifically investigate aneurysm size, but aneurysm location was evaluated and ultimately did not impact cost in the multivariate regression analysis.

\section{Cost-Containment Strategies}

Several approaches for cost containment in the treat- ment of aneurysms have been explored. One approach in reducing overall cost involves the appropriate selection of patients for postoperative monitoring in a surgical stepdown or acute care environment with closer monitoring to limit the facility costs. This method is problematic with aneurysm treatment because patients often require intensive care monitoring and treatment, especially in the

TABLE 2. Univariate and multivariable analysis of factors affecting cost

\begin{tabular}{lccccc}
\hline & \multicolumn{2}{c}{ Univariate } & & \multicolumn{2}{c}{ Multivariable } \\
\cline { 2 - 3 } \cline { 5 - 6 } \multicolumn{1}{c}{ Cost Factor } & $\begin{array}{c}\text { Standardized } \\
\beta\end{array}$ & $\begin{array}{c}p \\
\text { Value }\end{array}$ & & $\begin{array}{c}\text { Standardized } \\
\beta\end{array}$ & $\begin{array}{c}p \\
\text { Value }\end{array}$ \\
\hline Aneurysm location & -0.04 & 0.4 & & -0.03 & 0.5 \\
\hline Rupture status & -0.5 & 0.0001 & & -0.4 & 0.0001 \\
\hline Procedure type & 0.08 & 0.06 & & 0.3 & 0.0001 \\
\hline Sex & 0.04 & 0.4 & & 0.05 & 0.2 \\
\hline ASA grade & 0.4 & 0.0001 & 0.2 & 0.0001 \\
\hline $\begin{array}{l}\text { Discharge disposi- } \\
\quad \text { tion }\end{array}$ & 0.5 & 0.0001 & 0.3 & 0.0001 \\
\hline Yr of operation & 0.2 & 0.0001 & & 0.2 & 0.0001 \\
\hline
\end{tabular}

For the entire multivariable model: $R^{2}=0.65, p=0.0001$. 
setting of aneurysm rupture. Controlling the price of the coils/flow diverters is an important option in limiting the total costs as well; however, as mentioned previously, as the size of the aneurysm increases, more coils/flow diverters are needed, adding substantially to the total costs. Treatment cost by aneurysm size was not factored into our study and could be an area of future investigation. Interestingly, no study to date reports the superiority of a certain coil type over the other. ${ }^{13,16}$ Thus, selecting these materials based on their costs may be an alternative. Gandhoke and colleagues ${ }^{5}$ developed a capped-price model and showed that with capitation of endovascular materials, substantial budget control on supply costs can be achieved. Kashlan et al. ${ }^{8}$ reported significant budget saving with endovascular interventions when economical contrast media and standardized coil pricing were employed and when medical waste was reduced. Our institution utilizes a neurovascular price-control committee in which these issues are discussed and negotiated with vendors. Input from neurosurgeons, hospital administrators, and vendors is considered. This is likely a trend at most institutions.

\section{Limitations of the Study}

Several limitations of our study include its retrospective nature and the single-site perspective. Our institution uses both traditional open neurovascular as well as dual-trained endovascular/open neurovascular surgeons. However, the expertise, training, and facility here may differ from other institutions, limiting the generalizability of our study findings. Other institutions may show differing cost structure and treatment preferences, which may greatly affect the cost of care. Additional factors in the treatment of aneurysms in this study were not evaluated, such as aneurysm size, the complexity of postoperative care, and the need for additional surgical treatment, and these factors may be important because each patient presents with significant heterogeneity. Evaluation of treatment outcomes in terms of survival and durability of treatment may be a future investigation that can also better elucidate the value of aneurysm treatment.

\section{Conclusions}

With the advancement of imaging techniques, the rate of detection of intracranial aneurysms has increased. This has led to an increased rate of intervention for this condition to prevent its undesired sequelae. Surgical clipping and endovascular approaches (coil embolization and flow diversion) remain the most widely used modalities in the treatment of intracranial aneurysms. As their use continues to rise, the financial implications of these modalities have been a subject of interest for health care systems and medical providers attempting to delineate the hidden factors that drive their total costs and control them as much as possible while maintaining high-quality medical care. Facility utilization and supplies constitute the major factors for total costs in aneurysm treatment strategies. Recognizing these factors and controlling them are potential strategies to mitigate the total costs to patients and health care systems. Because neurosurgeons understand the disease, treatment, and outcomes of their patients, they are clearly positioned to develop and implement approaches and protocols to improve resource utilization..$^{17}$ This premise is the foundation of our analysis of cost drivers in aneurysm treatment, which will ultimately enable us to alter protocols, enact change, and track costs and outcomes.

\section{Acknowledgments}

We thank Kristin Kraus, MSc, for her editorial assistance and Jacob Kresser for assistance with the VDO database.

\section{References}

1. Bairstow P, Dodgson A, Linto J, Khangure M: Comparison of cost and outcome of endovascular and neurosurgical procedures in the treatment of ruptured intracranial aneurysms. Australas Radiol 46:249-251, 2002

2. Bekelis K, McGirt MJ, Parker SL, Holland CM, Davies J, Devin CJ, et al: The present and future of quality measures and public reporting in neurosurgery. Neurosurg Focus 39(6):E3, 2015

3. Colby GP, Lin LM, Paul AR, Huang J, Tamargo RJ, Coon AL: Cost comparison of endovascular treatment of anterior circulation aneurysms with the pipeline embolization device and stent-assisted coiling. Neurosurgery 71:944-950, 2012

4. Familiari P, Maldaner N, Kursumovic A, Rath SA, Vajkoczy $\mathrm{P}$, Raco A, et al: Cost comparison of surgical and endovascular treatment of unruptured giant intracranial aneurysms. Neurosurgery 77:733-743, 2015

5. Gandhoke GS, Pandya YK, Jadhav AP, Jovin T, Friedlander RM, Smith KJ, et al: Cost of coils for intracranial aneurysms: clinical decision analysis for implementation of a capitation model. J Neurosurg [epub ahead of print August 25, 2017; DOI: 10.3171/2017.3.JNS163149], 2017

6. Halkes PH, Wermer MJ, Rinkel GJ, Buskens E: Direct costs of surgical clipping and endovascular coiling of unruptured intracranial aneurysms. Cerebrovasc Dis 22:40-45, 2006

7. Karsy M, Brock AA, Guan J, Bisson EF, Couldwell WT: Assessment of cost drivers in transsphenoidal approaches for resection of pituitary tumors using the value-driven outcome database. World Neurosurg 105:818-823, 2017

8. Kashlan ON, Wilson TJ, Chaudhary N, Gemmete JJ, Stetler WR Jr, Dunnick NR, et al: Reducing costs while maintaining quality in endovascular neurosurgical procedures. J Neurosurg 121:1071-1076, 2014

9. Kawamoto K, Martin CJ, Williams K, Tu MC, Park CG, Hunter C, et al: Value driven outcomes (VDO): a pragmatic, modular, and extensible software framework for understanding and improving health care costs and outcomes. J Am Med Inform Assoc 22:223-235, 2015

10. Kim M, Park J, Lee J: Comparative cost analysis for surgical and endovascular treatment of unruptured intracranial aneurysms in South Korea. J Korean Neurosurg Soc 57:455459, 2015

11. Lee VS, Kawamoto K, Hess R, Park C, Young J, Hunter C, et al: Implementation of a value-driven outcomes program to identify high variability in clinical costs and outcomes and association with reduced cost and improved quality. JAMA 316:1061-1072, 2016

12. Maud A, Lakshminarayan K, Suri MF, Vazquez G, Lanzino G, Qureshi AI: Cost-effectiveness analysis of endovascular versus neurosurgical treatment for ruptured intracranial aneurysms in the United States. J Neurosurg 110:880-886, 2009

13. McDougall CG, Johnston SC, Gholkar A, Barnwell SL, Vazquez Suarez JC, Massó Romero J, et al: Bioactive versus bare platinum coils in the treatment of intracranial aneurysms: the MAPS (Matrix and Platinum Science) trial. AJNR Am J Neuroradiol 35:935-942, 2014 
14. Missios S, Bekelis K: Hospitalization cost after spine surgery in the United States of America. J Clin Neurosci 22:16321637,2015

15. Molyneux A, Kerr R, Stratton I, Sandercock P, Clarke M, Shrimpton J, et al: International Subarachnoid Aneurysm Trial (ISAT) of neurosurgical clipping versus endovascular coiling in 2143 patients with ruptured intracranial aneurysms: a randomised trial. Lancet 360:1267-1274, 2002

16. Molyneux AJ, Clarke A, Sneade M, Mehta Z, Coley S, Roy $\mathrm{D}$, et al: Cerecyte coil trial: angiographic outcomes of a prospective randomized trial comparing endovascular coiling of cerebral aneurysms with either cerecyte or bare platinum coils. Stroke 43:2544-2550, 2012

17. Rosenbaum L, Lamas D: Cents and sensitivity-teaching physicians to think about costs. N Engl J Med 367:99-101, 2012

18. Simon SD, Reig AS, James RF, Reddy P, Mericle RA: Relative cost comparison of embolic materials used for treatment of wide-necked intracranial aneurysms. J Neurointerv Surg 2:163-167, 2010

19. Smith M, Saunders R, Stuckhardt L, McGinnis JM (eds): Best Care at Lower Cost: the Path to Continuously
Learning Health Care in America. Washington, DC: National Academies Press, 2013

\section{Disclosures}

Dr. Taussky reports being a consultant for Medtronic.

\section{Author Contributions}

Conception and design: Couldwell, Taussky. Acquisition of data: Twitchell, Reese, Karsy. Analysis and interpretation of data: Karsy. Drafting the article: Twitchell, Abou-Al-Shaar, Reese, Karsy. Critically revising the article: Couldwell, Abou-Al-Shaar, Reese, Karsy, Eli, Guan, Taussky. Reviewed submitted version of manuscript: Couldwell, Twitchell, Reese, Karsy, Eli, Guan, Taussky. Approved the final version of the manuscript on behalf of all authors: Couldwell.

\section{Correspondence}

William T. Couldwell: University of Utah, Salt Lake City, UT. neuropub@hsc.utah.edu. 